\title{
ORT_23 - Development of human angiotensin converting enzyme-2 (hACE2) as a strategy to face COVID-19
}

Haroldo Cid da Silva Junior ${ }^{1}$; Fernanda Otaviano Martins ${ }^{1}$; Cristiane Pinheiro Pestana ${ }^{1}$; Janaina Figueira Mansur ${ }^{1}$; Ana Paula Corrêa Argondizzo ${ }^{1}$; Mariana Miguez Tardelli Garcia ${ }^{1}$; Gabriela dos Santos Esteves ${ }^{1}$. ${ }^{1}$ Fiocruz/Bio-Manguinhos.

Introduction: The rapid worldwide spread of SARS-CoV-2 leads to an urgent need for prophylactic and therapeutic measures to combat COVID-19. Angiotensin Converting Enzyme-2 (ACE2) is a monocarboxypeptidase, component of the renin-angiotensin system and its extracellular domain has been demonstrated as a receptor for the spike (S) protein of SARS-CoV-2. This enzyme is barely present in the circulation, but widely expressed in organs, such as the kidneys and the gastrointestinal tract, with relatively low level of expression in lungs. The development of therapeutic agents that block specifics points of the virus replication pathway like the interaction between the viral spike glycoprotein and hACE2 on host cells, could be a promising strategy.

Objective: To express and purify hACE2-IgG1-Fc fusion protein using Expi293 cell system.

Methodology: The coding sequence of hACE2-IgG1-Fc fusion protein was cloned into pcDNA3.4 vector by a custom gene synthesis service and used to transfect mammalian Expi293 cells. The protein expression was evaluated by SDS-PAGE under reduction condition and its identity was confirmed by western blotting using AP-anti-IgG antibody (heavy and light chain). Then, hACE2-IgG1-Fc was purified by affinity chromatography using A protein from Staphylococcus aureus as the ligand and further analyzed by densitometry to estimate homogeneity level. In order to evaluate ACE2 and S1 SARS-CoV-2 interaction, the S1 protein (GenBank - protein ID: QHO62112.1) was also expressed in Expi293 cells and purified by immobilized metal?ion affinity chromatography (IMAC).

Results: The hACE2-IgG1-Fc was detected in supernatant of Expi293 cells after transfection assay. A band around $150 \mathrm{kDa}$ was observed at the SDS-PAGE analysis, which corresponds to the molecular weight expected to its monomeric form. The hACE2-IgG1-Fc was successfully purified after a single step chromatography with $95 \%$ of purity. In parallel, S1 SARS-CoV-2 was obtained with $85 \%$ purity. This protein contains receptor binding domain $(\mathrm{RBD})$ of virus and will be used to further interaction studies involving hACE2-IgG-Fc.

Conclusion: Obtaining hACE2-IgG1-Fc with high yield and purity will make it possible to carry out in vitro neutralization tests and evaluation of its therapeutic and prophylactic potential in animal models of SARS-CoV-2 infection.

Keywords: COVID-19; ACE2; SARS-CoV-2 